\title{
THORSTEN BRANTS
}

\section{Statistisch basierte Sprachmodelle und maschinelle Übersetzung}

\begin{abstract}
Statistische Methoden finden derzeit in der Sprachtechnologie vielfache Verwendung. Ein Grundgedanke dabei ist das Trainieren von Programmen auf großen Mengen von Daten. Für das Trainieren von statistischen Sprachmodellen gilt zur Zeit das Motto ,Je mehr Daten desto besser". In unserem System zur maschinellen Übersetzung sehen wir eine fast konstante qualitative Verbesserung (gemessen als BLEU-Score) mit jeder Verdoppelung der monolingualen Trainingsdatenmenge. Selbst bei Mengen von ca. 20 Milliarden Wörtern aus Nachrichtentexten und ca. 200 Milliarden Wörtern aus Webseiten ist kein Abflachen der Lernkurve in Sicht.

Dieser Artikel gibt kurze Einführungen in statistische maschinelle Übersetzung, die Evaluation von Übersetzungen mit dem BLEU-Score, und in statistische Sprachmodelle. Wir zeigen, welch starken Einfluß die Größe der Trainingsdaten des Sprachmodells auf die Übersetzungsqualität hat. Danach wird die Speicherung großer Datenmengen, das Trainieren in einer parallelen Architektur und die effiziente Verwendung der bis zu 1 Terabyte großen Modelle in der maschinellen Übersetzung beschrieben.
\end{abstract}

\section{Statistische maschinelle Übersetzung}

Dieses Feld findet in den letzten Jahren mehr und mehr Interesse in der Forschung. Aufgrund der weltweit steigenden Verbreitung des Internets besteht auch ein steigender Bedarf an Übersetzungen von Texten, um Webseiten auch denjenigen Benutzern zugänglich zu machen, die die Originalsprache des Dokuments nicht verstehen. Leider lässt die Übersetzungsqualität der existierenden Systeme oft noch sehr viel zu wünschen übrig. Im Folgenden ein Beispiel für eine Übersetzung aus dem Arabischen ins Englische, bei dem die geringe Qualität ein Verstehen fast unmöglich macht:

The Bradi : The inspectors need to "a few months" for end important their

Paris $13-1$ (aa so in in) - the general manager for agency announced international for energy atomic Mohammed the Bradi today Monday that inspectors of international nze' the weapons need to "a few months" for end important their in Iraq.

Journalistic conference in end of meeting with French External Minister of Dominique de Villepin that the inspectors said during "a few their need to important months for end".

Said that he not can to the accurate for conclusion schedule an appointment important the inspectors and definitive importance the appointment lessened from on January 27 
indicative to that the report who Security Council at that the date of he will hand over to "temporary report".

The Bradi that Security Council confirmed “ünderstands" that January 27 final term not.

Als positives Beispiel hier die Übersetzung, die unser Google Research System vom gleichen Originaltext produziert:

\section{ElBaradei : Inspectors Need "a Few Months" to Complete Their Mission}

Paris 13 - 1 (AFP) - The Director General of the International Atomic Energy Agency Mohamed ElBaradei today, Monday, that the international disarmament inspectors need "a few months" to complete their mission in Iraq.

He said during a press conference at the conclusion of a meeting with French Foreign Minister Dominique de Villepin that the inspectors "need a few months to complete their mission."

He could not specify the exact date of the conclusion of the task of the inspectors and reduced the importance of the deadline of 27 January, pointing out that the report will be handed to the Security Council on that date is a "progress report".

ElBaradei stressed that the Security Council "ünderstands" that the 27 January deadline is not final.

Auch ohne den Originaltext zu kennen, lässt sich unschwer erkennen, dass dies eine bessere Übersetzung ist. Bis auf einige kleinere Fehler, die das Verstehen nur wenig einschränken (z. B. Fehlen des Verbs ,said“ im ersten Satz), hat der übersetzte Text in etwa die Qualität eines von einem Muttersprachler produzierten Textes.

Statistische maschinelle Übersetzungssysteme kombinieren in der Regel mindestens zwei statistische Modelle: das Übersetzungsmodell und das Sprachmodell (Brown et al. 1990). Das Übersetzungsmodell liefert Wahrscheinlichkeiten $p(f \mid e)$ dafür, dass einzelne Wörter oder relative kurze Sequenzen von Wörtern in der Quellsprache $f$ Übersetzungen von Wörtern oder Sequenzen in der Zielsprache $e$ sind. ${ }^{1}$ Aufgrund dessen werden Satzbruchstücke als Kandidaten für Übersetzungen ausgewählt. Das Sprachmodell liefert Wahrscheinlichkeiten $p(e)$ für Sequenzen von Wörtern in der Zielsprache $e$. Diese sorgen dafür, dass die Kandidatenbruchstücke in die richtige Reihenfolge gebracht werden. Die meisten Systeme verwenden zusätzlich weitere Informationsquellen (Features) wie z. B. die Satzlänge, wie viele verschiedene Bruchstücke verwendet werden, wie stark die Reihenfolge in der Zielsprache von der Reihenfolge in der Ausgangssprache abweicht usw. Alle verwendeten statistischen Modelle und weiteren Features werden dann in einem log-linearen Model miteinander kombiniert:

$$
\log (\text { score })=\lambda_{1} \log (p(f \mid e))+\lambda_{2} \log (p(e))+\lambda_{3} \ldots
$$

1 Die Abkürzungen $e$ und $f$ standen ursprünglich für english als Zielsprache und french als Quellsprache, da ein Großteil der frühen Arbeiten zur statistischen maschinellen Übersetzung sich mit diesem Sprachpaar beschäftigte. Jetzt werden sie unabhängig vom Sprachpaar weiterverwendet. 
Die Übersetzungswahrscheinlichkeiten $p(f \mid e)$ und die meisten anderen Features sowie deren Gewichtungsparameter $\lambda$ werden anhand von parallelen Trainingsdaten gelernt, d.h. anhand von Dokumenten, die Übersetzungen voneinander sind. Im phrasenbasierten Ansatz wird versucht, innerhalb von Sätzen möglichst lange Phrasen zu identifizieren, die häufig Übersetzungen voneinander sind (Och et al. 1999; Koehn et al. 2003). Dabei sind mit Phrasen in der Regel keine Phrasen im linguistischen Sinne gemeint, sondern beliebige Sequenzen von Wörtern, Zahlen und Satzzeichen, die häufig gemeinsam mit einer bestimmten Sequenz in der anderen Sprache auftreten.

Die Sprachmodell-Wahrscheinlichkeiten $p(e)$ werden anhand von monolingualen Daten gelernt. Hierfür stehen die größten Datenmengen bereit. Dies wird weiter unten eingehender betrachtet.

\section{Qualitätsbestimmung durch BLEU-Scores}

Traditionell wird die Qualität von Übersetzungen durch menschliche Evaluation festgestellt. Möglich sind z. B. das Bewerten von Übersetzungen auf einer mehrstufigen Skala für verschiedene Kategorien wie Adäquatheit oder Flüssigkeit (adequacy, fluency) oder der direkte Vergleich zweier verschiedener Übersetzungen, indem ein Annotierer bestimmt, ob Übersetzung A oder B die bessere ist. Menschliche Evaluation erlaubt sehr sichere Qualitätsurteile und ist damit auch heute noch die zuverlässigste Methode für Evaluationen. Sie hat jedoch den Nachteil, dass sie sehr zeit- und arbeitsaufwendig ist und damit nur schlecht für Entwicklungszyklen automatischer Systeme geeignet ist.

Papineni et al. (2002) stellten daher ein automatisches System mit dem Namen „BLEU“ vor (oder auch: BLEU-Score). Dieses System benötigt ebenfalls menschliche Übersetzer, jedoch lässt es von diesen nur genau einmal (vor der eigentlichen Evaluation) mehrere Übersetzungen des gleichen Texts anfertigen und vergleicht dann automatisch, wie nah die zu evaluierenden Übersetzungen den Referenzübersetzungen sind. Sie zeigten in ihrer Studie, dass dieser automatische Vergleich sehr stark mit menschlichen Urteilen korreliert, wenn eine große Zahl von Testsätzen verwendet wird, und dass in vielen Fällen sowohl menschliche als auch automatische Evaluation zur gleichen Rangreihenfolge der zu vergleichenden Übersetzungen führt.

Der BLEU-Score gab der Forschung zur maschinellen Übersetzung neuen Antrieb. Evaluationen, die bisher Wochen oder gar Monate dauerten, finden nun innerhalb weniger Sekunden statt. Die Idee hinter dem BLEU-Score wird in dem folgenden Beispiel erläutert. Wir nehmen an, dass es drei menschliche englische Referenzübersetzungen eines Textes gibt:

Referenz 1: Japan remained the biggest trading partner

Referenz 2: Japan is still the largest trade partner

Referenz 3: Japan still remains the number one trade partner 
Jetzt soll bestimmt werden, welches von drei verschiedenen Systemen die beste Übersetzung erstellt. Der BLEU-Score zieht Wortsequenzen (N-gramme) der Längen 1 bis 4 in Betracht ${ }^{2}$, die mit den Referenzübersetzungen übereinstimmen. Alle Übereinstimmungen sind im folgenden hervorgehoben:

\section{System 1: Japan will continue to be partner big}

System 2: Japan is still the biggest trading partner

System 3: Ukraine won 2:1 against Poland

System 1 generierte lediglich zwei übereinstimmende Unigramme: „Japan“ und „partner“. Die Übersetzung von System 2 ist komplett durch $\mathrm{N}$-gramme in den Referenzen abgedeckt: durch „Japan is still the“ aus Referenz 1, und durch „the biggest trading partner" aus Referenz 2. System 3 generierte keines der Referenzworte. Je mehr Text durch übereinstimmende N-gramme abgedeckt ist und je länger die N-gramme sind, desto besser die Qualität. Demnach lieferte System 2 die beste Übersetzung. Dies deckt sich sehr gut mit menschlichen Urteilen.

Der BLEU-Score verwendet:

- Die modifizierte N-gramm-Präzision: Für die Standard N-gramm-Präzision wird errechnet, welcher Anteil der maschinell erzeugten N-gramme der Länge $\mathrm{k}$ auch in den Referenzübersetzungen erscheint. Die modifizierte $\mathrm{N}$-gramm-Präzision lässt nur die einmalige Benutzung jedes Teils der Referenzübersetzung zu.

- Brevity Penalty: Dieser Parameter bestraft die Erstellung von zu kurzen Übersetzungen.

- BLEU-4-Score: Das geometrische Mittel der N-gramm-Präzision der Längen $\mathrm{N}=1$ bis 4 , multipliziert mit der Brevity Penalty.

Bei großen Mengen von Sätzen besteht normalerweise eine hohe Korrelation zwischen dem BLEU-Score und menschlichen Urteilen zur Qualität der Übersetzung. Die BLEU-Score-Werte wachsen mit der Anzahl der Referenzübersetzungen. Beispielsweise erreichen die besten statistischen Systeme bei der Übersetzung von Nachrichtentexten vom Arabischen ins Englische BLEU-Scores von mehr als 50\%, wenn 4 Referenzübersetzungen verwendet werden. Zum Vergleich: Menschliche Übersetzungen erreichen Werte zwischen 50 und $60 \%$ (leicht über statistischen Systemen), und die existierenden kommerziellen (nicht-statistischen) System erreichen lediglich Werte von 10 bis $35 \%$. Die BLEU-Scores für Übersetzungen vom Chinesischen ins Englische liegen insgesamt niedriger (Werte in den 30ern), weisen aber ähnliche Muster auf.

Das amerikanische National Institute of Standards and Technology (NIST) führt jährlich eine Evaluation von automatischen Übersetzungssyste-

2 Dies ist der sogenannte BLEU-4-Score, der am häufigsten verwendet wird. Andere Maximallängen sind möglich geben jedoch in der Regel sehr ähnliche Ergebnisse. 
men durch. Im Jahre 2005 erreichte das Google Research System BLEU-Scores von 51,37\% für Arabisch-Englisch und 35,31\% für Chinesisch-Englisch. Wir waren mit diesen Werten sehr zufrieden. Die Ergebnisse der anderen Teilnehmer sind auf den Webseiten von NIST zu finden. ${ }^{3}$

\section{Statistische Sprachmodelle}

Ein Standard-Ansatz für statistische Sprachmodelle ist die Verwendung von $\mathrm{N}$-grammen, deren Wahrscheinlichkeiten aufgrund ihrer Auftretenshäufigkeiten in größeren Textmengen geschätzt werden. Die erste große Anwendung für diese Modelle war die automatische Spracherkennung (Jelinek/Mercer 1980). N-gramme sind Sequenzen von $N$ Tokens, wobei ein Token aus einem Wort, einer Zahl oder Interpunktion besteht. Bei Verwendung von N-grammen wird die Wahrscheinlichkeit des nächsten Wortes bestimmt unter der Bedingung, dass die vorherigen $N-1$ Wörter bekannt sind. Die einzelnen Wahrscheinlichkeiten werden gemäß der Kettenregel multipliziert. Lautet z. B. die zu verarbeitende Wortfolge „NASA officials say they hope ...“, dann ergibt sich dessen Wahrscheinlichkeit in einem Trigramm-Modell $(N=3)$ aus

$$
\begin{aligned}
& p \text { (NASA officials say they hope } \ldots) \\
& =p \text { (NASA) } p \text { (officials | NASA) } p \text { (say | NASA officials }) \\
& \quad p \text { (they } \mid \text { officials say) } p \text { (hope } \mid \text { say they) } p(\ldots \mid \text { they hope })
\end{aligned}
$$

oder allgemein für einen Satz mit den Wörtern $w_{1} \ldots w_{k}$ :

$$
p\left(w_{1}, \ldots, w_{\mathrm{k}}\right)=\prod_{i=1}^{k} p\left(w_{i} \mid w_{i-N+1}, \ldots, w_{i-1}\right)
$$

N-gramm-Modelle sind auch unter der Bezeichnung Markov-Modell bekannt (Markov 1913). Die Wörter $w_{i-N+1} \ldots w_{i-1}$ bilden den vorherigen Kontext (auch Geschichte, History), $w_{i}$ ist das nächste Wort. Die Wahrscheinlichkeiten auf der rechten Seite der Gleichung werden anhand von relativen Häufigkeiten geschätzt,

$$
p\left(w_{i} \mid w_{i-\mathrm{N}+1}, \ldots, w_{i-1}\right)=\frac{f\left(w_{i-\mathrm{N}+1}, \ldots, w_{i}\right)}{f\left(w_{i-\mathrm{N}+1}, \ldots, w_{i-1}\right)}
$$

wobei $f$ die Häufigkeit im zugrunde liegenden Korpus repräsentiert. Im konkreten Fall ist dies zum Beispiel für die Wahrscheinlichkeit, dass das nächste Wort „say“ ist, wenn der vorherige Kontext „NASA officials“ war:

$$
p(\text { say } \mid \text { NASA officials })=\frac{f(\text { NASA officials say })}{f(\text { NASA officials })}=\frac{752}{12819}=0.05866
$$

3 (http://www.nist.gov/speech/tests/mt/mt05eval_official_results_release_20050801_ v3.html 


\section{Glättung}

Die direkte Verwendung von relativen Häufigkeiten führt in der Regel zu Problemen mit Null-Wahrscheinlichkeiten, falls eines der beteiligten $\mathrm{N}$-gramme nicht im Korpus auftritt. Diese sorgen dafür, dass die Wahrscheinlichkeit eines gesamten Satzes ebenfalls Null wird, da die einzelnen Wahrscheinlichkeiten multipliziert werden. Damit würde allen Sätzen, die eine solche NullWahrscheinlichkeit enthalten, die gleiche Wahrscheinlichkeit von Null zugewiesen, und wir könnten keine Aussage mehr darüber machen, welcher Satz der bessere oder schlechtere ist. Um dies zu vermeiden, wird Glättung (Smoothing) eingesetzt. Chen und Goodman (1998) geben eine umfassende Übersicht zu diesem Thema. Standardverfahren sind das Backoff und die gewichtete Interpolation. Im Backoff werden kürzere $\mathrm{N}$-gramme verwendet, falls der vorherige Kontext seltener als ein vorbestimmter Schwellwert auftritt:

$$
p\left(w_{i} \mid w_{i-n+1}, \ldots, w_{i-1}\right)= \begin{cases}\hat{p}\left(w_{i} \mid w_{i-n+1}, \ldots, w_{i-1}\right) & \text { falls } f\left(w_{i-n+1}, \ldots, w_{i}\right)>\theta \\ \lambda p\left(w_{i} \mid w_{i-n+2}, \ldots, w_{i-1}\right) & \text { sonst }\end{cases}
$$

In der gewichteten Interpolation werden ebenfalls längere und kürzere Ngramme gemeinsam verwendet, allerdings gewichtet unter Verwendung eines Faktors $\lambda$ :

$$
p\left(w_{i} \mid w_{i-N+1}, \ldots, w_{i-1}\right)=\lambda \hat{p}\left(w_{i} \mid w_{i-N+1}, \ldots, w_{i-1}\right)+(1-\lambda) p\left(w_{i} \mid w_{i-N+2}, \ldots, w_{i-1}\right)
$$

Dies ist eine rekursive Definition, d.h. die Wahrscheinlichkeit des kürzeren $\mathrm{N}$-gramms im rechten Teil der Gleichung wird wiederum durch eine gewichtete Interpolation mit dem noch kürzeren $\mathrm{N}$-gramm bestimmt.

\section{Korpora}

Für das Trainieren eines Sprachmodells benötigen wir große Mengen an Daten in der Zielsprache. Wir benutzten unter anderem die Gigaword Corpora in Englisch, Chinesisch und Arabisch, die vom Linguistic Data Consortium (LDC) herausgegeben werden. Sie enthalten jeweils einige 100 Millionen bis einige Milliarden Wörter pro Sprache. Als sich im Laufe unserer Arbeit an dem System der Bedarf an mehr und mehr Daten ergab, begannen wir, Google-Daten aus dem Internet zu benutzen. Dies sind zum einen Nachrichtentexte aus dem „Google News“ Service. Im aktuellen System verwenden wir davon ca. 25 Milliarden Wörter englischer Texte und 17 Milliarden Wörter chinesischer Texte. Für das Deutsche stehen ca. 4 Milliarden Wörter aus Nachrichtentexten bereit. Weitere Sprachen wie z. B. Französisch, Italienisch, Spanisch, Arabisch und andere sind ebenfalls vorhanden. Nachrichtentexte haben den Vorteil, dass öffentlich zugängliche Daten dieser Domäne täglich wachsen und ständig neue Ereignisse durch sie abgedeckt werden. Weiterhin verwenden wir Texte aus allgemeinen Web-Seiten, die in noch größeren Mengen zur Verfügung stehen. So verarbeiteten wir z. B. 200 Milliarden Wörter aus englischen Seiten für unser aktuelles Übersetzungssystem. Daten für an- 
dere Sprachen stehen ebenfalls zur Verfügung, z. B. ca. 88 Milliarden Wörter für das Chinesische und ca. 30 Milliarden Wörter für das Deutsche. Dieses sind nur kleine Bruchstücke der insgesamt bei Google indizierten Web-Daten, und wir arbeiten daran, noch größere Mengen für unser Übersetzungssystem zu verwenden.

Zum Trainieren des Sprachmodells in unserem System zur maschinellen Übersetzung werden unannotierte Daten verwendet, die nur einer geringfügigen Vorverarbeitung unterzogen werden (z. B. Tokenisierung und SatzendeErkennung). Dies ist eine wichtige Voraussetzung. Der Einsatz manueller Schritte würde die Verwendung solch großer Datenmengen unmöglich machen. Weitere Gebiete, die ebenfalls von großen Mengen unannotierter Daten profitieren können, sind unter anderen die automatische Spracherkennung, Texterkennung und die Rechtschreibkorrektur.

\section{Einfluss der Sprachmodellierung auf die Übersetzungsqualität}

„Je mehr Daten desto besser“ ist das Motto, das hier überprüft werden soll. Dafür trainierten wir 5-gramm-Sprachmodelle auf verschiedenen Mengen von Nachrichtentexten und integrierten diese in unser Übersetzungssystem. Alle auftretenden $\mathrm{N}$-gramme wurden verwendet, d.h. die Mindesthäufigkeit für N-gramme im Modell war 1. Für die Glättung wurde ein Backoff-Verfahren angewandt. Die kleinste Datenmenge ist 75 Millionen Wörter. Danach wird die Datenmenge in jedem Schritt in etwa verdoppelt, insgesamt achtmal, bis 18 Millionen Wörter erreicht sind. Getestet wird auf den Daten der NIST MT Evaluation von 2003 für Übersetzungen aus dem Arabischen ins Englische. Die Testdaten umfassen 100 Texte mit insgesamt 663 Sätzen. ${ }^{4}$ Die Ergebnisse sind in Abbildung 1 dargestellt.

Wir sehen eine fast konstante Verbesserung von ca. $0.5 \%$ im BLEU-Score bei jeder Verdoppelung der Datenmenge. Dies ist eine sehr deutliche Verbesserung, und viele in der Literatur beschriebenen Neuerungen liefern kleinere Verbesserungen des BLEU-Scores. Auch bei 18 Milliarden Wörtern scheint die Kurve nicht abzuflachen. ${ }^{5}$

Schließlich fügten wir noch ca. 200 Milliarden Wörter aus Webseiten zum Sprachmodell hinzu. Die Testdaten bestehen im wesentlichen aus Nachrichtentexten. Die Verwendung einer anderen Domäne lässt geringere Verbesserungen erwarten. Dies bestätigte sich auch in unseren Untersuchungen. Insgesamt ergibt der Sprung von 18 Milliarden Wörtern Nachrichtentexten auf 200 Milliarden Wörtern aus Webtexten eine Verbesserung von $0.9 \%$. Dies

4 Diese Daten sind vom Linguistic Data Consortium unter der Katalognummer LDC2005T05 erhältlich.

518 Millionen Wörter ist die größte Menge an Nachrichtentexten die zum Zeitpunkt der Experimente zur Verfügung stand. Inzwischen liegen mehr Daten vor und es wird interessant zu sehen, wie die Kurve weiter verläuft. 


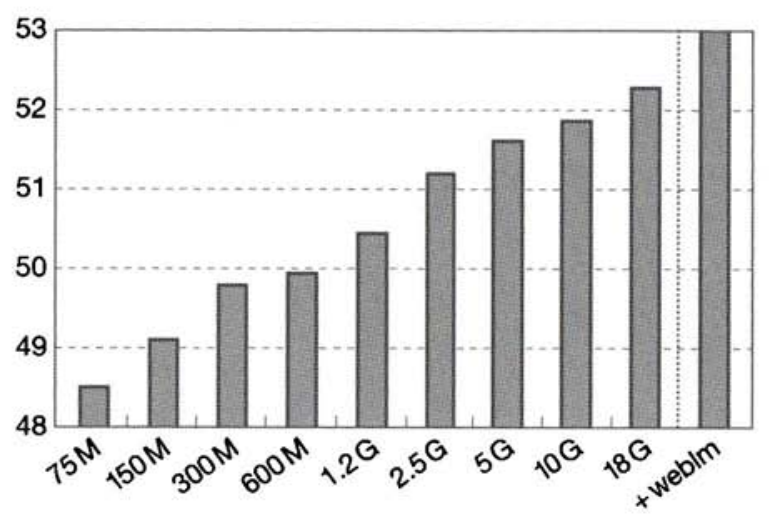

Abb. 1: BLEU-Scores für NIST 2003 MT Evaluationsdaten Arabisch-Englisch bei Verwendung verschiedener Mengen von Nachrichtentexten für das Sprachmodell ( $\mathrm{M}=$ Millionen, $\mathrm{G}=$ Milliarden Wörter).

entspricht immerhin noch einer Steigerung von ca. $0.25 \%$ bei jeder Verdoppelung der Datenmenge. Webtexte sind zwar weniger hilfreich für unsere Testdaten, liegen jedoch nur genügend davon vor, dann ist trotzdem eine deutliche Steigerung der Übersetzungsqualität sichtbar.

Ähnliche Untersuchungen mit Mengen paralleler Daten ergaben, dass jede Verdopplung der Datenmenge den BLEU-Score um ca. $2.5 \%$ verbessert. Der Einfluss paralleler Daten ist somit deutlich höher als der monolingualer Daten. Jedoch liegen letztere in viel größeren Mengen vor. Die vom Linguistic Data Consortium vertriebenen parallelen Korpora haben 100-200 Millionen Wörter für die größten Sprachpaare. Die in dieser Untersuchung verwendeten monolingualen Datenmengen sind um den Faktor 1000 größer.

Das auf 200 Milliarden Wörtern trainierte Sprachmodell ist riesig. Es enthält 45,6 Milliarden $\mathrm{N}$-gramme, und die zugehörigen Tabellen benötigen ca. 1.1 Terabyte Speicherplatz. $66,5 \%$ der N-gramme sind Singletons, d. h., treten nur einmal auf. Jedoch schadet das Entfernen der Singletons der Übersetzungsqualität leicht. Im folgenden betrachten wir das Trainieren und die Anwendung dieser Modelle.

\section{Verteiltes Trainieren von Sprachmodellen}

Die Rohtexte werden verteilt in mehreren hundert Dateien im Google File System (GFS) gespeichert (Ghemawat et al. 2003). Dies ist ein Dateisystem, das auf Standardhardware läuft und jeden Datenblock auf drei verschiedenen Maschinen repliziert. Ausgefallene Maschinen werden automatisch erkannt, und die Software erstellt neue Kopien von Datenblöcken, so dass immer drei Kopien vorhanden sind. Das zugrunde liegende Netzwerk besteht aus mehr als 1000 Computern mit insgesamt mehr als 300 Terabyte Plattenspeicherplatz. Dieses dient als Basis für die parallele Verarbeitung der Daten. 
Als Software-Infrastruktur dient MapReduce (Dean/Ghemawat 2004). Dieses parallelisiert die Verarbeitung automatisch. Die Daten werden unterteilt in Blöcke (die Shards), auf denen die Verarbeitung in zwei Phasen abläuft. Phase 1 (,Map“) bildet die Eingabedaten auf temporäre Schlüssel-WertePaare ab. Jeder Schlüssel wird dann aufgrund einer Hashfunktion einem Shard für Phase 2 („Reduce“) zugewiesen. Diese Phase kombiniert alle temporären Paare mit dem gleichen Schlüssel und schreibt dann die Ausgaben.

Im ersten Schritt unserer Verarbeitung erstellen wir das Vokabular. In diesem konkreten Fall eines MapReduce dienen die Wörter als Schlüssel und deren Häufigkeiten als Werte. Die temporären Paare enthalten die Wörter und deren Häufigkeiten für einen bestimmten Teil der Eingabedaten (z. B. für eine der mehreren hundert Dateien). Alle Paare für dasselbe Wort werden zum selben „Reduce“-Shard geschickt, in dem dann die Häufigkeiten addiert werden. Schließlich wird das Wort zusammen mit der Summe der Häufigkeiten in die Ausgabedatei des entsprechenden Shards geschrieben. Wörter, die zu selten auftreten, z.B. seltener als einmal in 5 Milliarden Wörtern, werden entfernt. An ihre Stelle tritt das ,unbekannte Word“ (<UNK $>$ ). Damit müssen später weniger $\mathrm{N}$-gramme gespeichert werden, da z. B. die Häufigkeiten $f(\mathrm{~A}$ B SELTENES_WORT_1 C) und $f($ A B SELTENES_WORT_2 C) denselben Eintrag belegen, nämlich $f(\mathrm{~A} \mathrm{~B}<\mathrm{UNK}>\mathrm{C}$ ). Für alle anderen Wörter werden im nächsten Schritt direkt die N-gramme gesammelt. Als Beispiel für den Einfluss der Minimalhäufigkeiten auf die Datengröße geben wir hier Größen an, die sich bei der Bearbeitung von 200 Milliarden Wörtern aus Webdaten ergaben: bei einer minimalen Worthäufigkeit von 1 werden 400 Millionen verschiedene „Wörter“ gefunden, was zu 1.1 Terabyte 5-gramm-Daten führt, während bei einer minimalen Häufigkeit von 40 lediglich 11 Millionen verschiedene „Wörter“ verbleiben, was zu 800 Gigabyte 5-gramm-Daten führt.

Die Vokabularerstellung ist in Abbildung 2 schematisch dargestellt mit zwei Reduce-Shards. Tatsächlich verwenden wir 100 und mehr Shards für diesen Schritt. Abbildung 3 zeigt ein sehr vereinfachtes Beispiel mit drei Dokumenten. Die tiefgestellten Indizes vor den N-grammen zeigen den Wert der Hash-

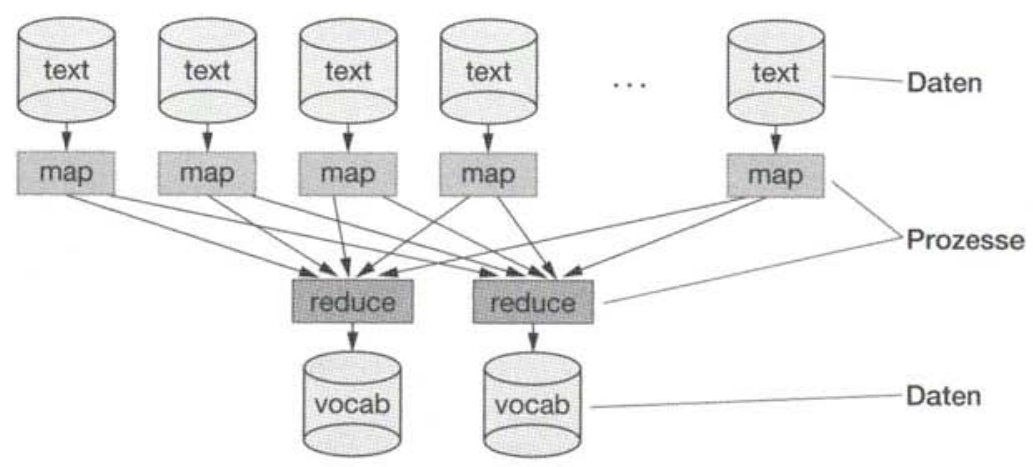

Abb. 2: Schematische Darstellung der Vokabularerstellung als MapReduce. 


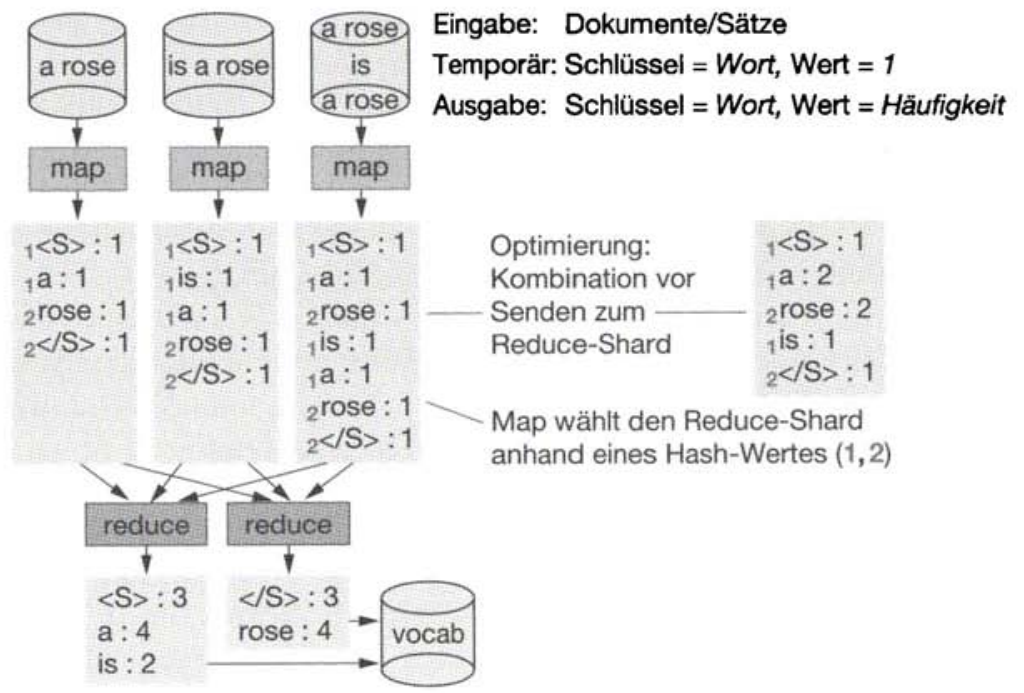

Abb. 3: Beispiel für die Vokabularerstellung mit drei Eingabedokumenten.

funktion, die bestimmt, zu welchem Reduce-Shard das N-gramm geschickt wird.

Im zweiten Schritt werden die eigentlichen N-gramm-Tabellen zusammengestellt. Hier kommt ebenfalls MapReduce zum Einsatz. Jetzt sind die $\mathrm{N}$-gramme die Schlüssel, die Häufigkeiten sind wiederum die Werte. Die Anzahl der Map- und Reduce-Shards ist jetzt viel größer um die viel größere Menge der $\mathrm{N}$-gramme zu verarbeiten. Typischerweise verwenden wir hier 1000 Shards. Ein sehr einfaches Beispiel für die Erstellung von Bigrammen aus drei Dokumenten ist in Abbildung 4 dargestellt. Für dieses Beispiel wurden den Dokumenten zwei Wörter hinzugefügt, die jeweils nur einmal auftreten, um auch die Verwendung von $<\mathrm{UNK}>$ zu illustrieren.

Im nächsten Schritt wird die relative Häufigkeit $r$ eines jeden $\mathrm{N}$-gramms bestimmt. Diese ergibt sich aus der Häufigkeit $f$ des $\mathrm{N}$-gramms und der des linken Kontexts:

$$
r(\mathrm{E} \mid \mathrm{ABCD})=\frac{f(\mathrm{ABCDE})}{f(\mathrm{ABCD})}
$$

Ein Problem dabei ist, dass die beiden Häufigkeiten $f$ nicht notwendigerweise im selben Shard gespeichert sind, auf diese aber gleichzeitig zugegriffen werden muss. Ein wahlfreier Zugriff auf die auf Festplatte gespeicherten Tabellen wäre sehr langsam. Eine Lösung dafür ist, dass die gewählte HashFunktion $\mathrm{N}$-gramme aufgrund ihres ersten Wortes verteilt. Im Beispiel oben wären damit alle $\mathrm{N}$-gramme, die mit dem Wort „A“ beginnen, im selben Shard. Relative Häufigkeiten können so in nur einem Lauf über den betreffenden Shard berechnet werden. Ein Nachteil dieses Verfahrens ist, dass 


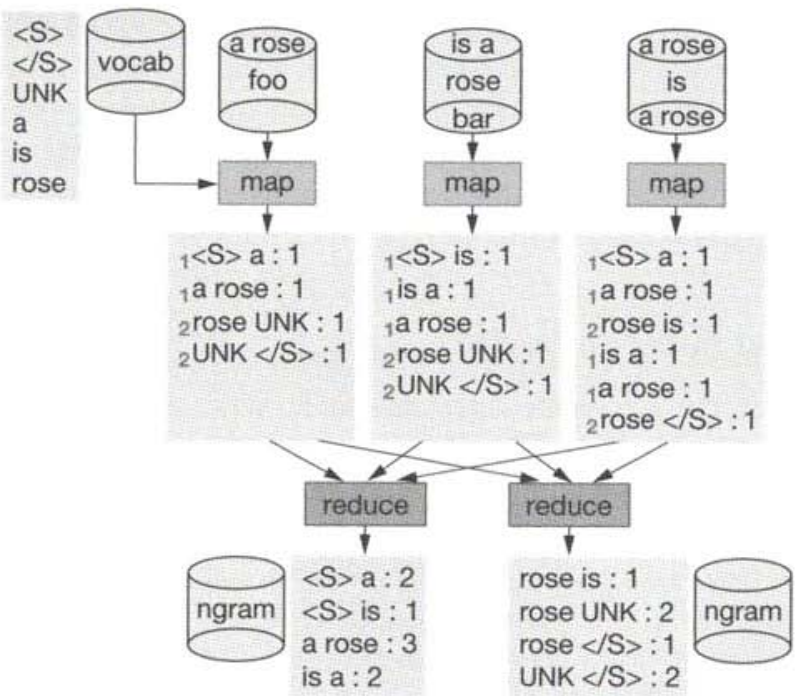

Abb. 4: Beispiel für die N-gramm-Erstellung mit drei Eingabedokumenten.

einige Wörter (z. B. Funktionswörter) sehr viel häufiger sind als andere. Shards, die diese sehr häufigen Wörter speichern, werden in der Regel viel größer als andere Shards. Dies möchten wir vermeiden, damit die Verarbeitungszeiten für alle Shards in etwa gleich sind. Die ungleichmäßige Verteilung kann in den meisten Fällen dadurch gelöst werden, dass die Hash-Funktion die ersten zwei Wörter berücksichtigt und alle Unigramme in allen Shards repliziert werden (damit relative Häufigkeiten von Bigrammen ebenfalls innerhalb eines Shards berechnet werden können). Dies wird Präfix-Sharding genannt. Die Vervielfältigung der Unigramme hat nur einen sehr kleinen Einfluss auf den benötigten Speicherplatz, da ihre Anzahl viel kleiner ist als die der N-gramme höherer Ordnung.

Der letzte Schritt für die Erstellung des Sprachmodells ist das Smoothing. Dafür muss auf kürzere $\mathrm{N}$-gramme zugegriffen werden, die durch Entfernen des jeweils ersten Wortes im linken Kontext entstehen. So muss z.B. zur Berechnung der Wahrscheinlichkeit eines 5-gramms $p(E \mid A B C D)$ im Smoothing auf diese fünf relativen Häufigkeiten zugegriffen: $r(E \mid A B C D), r(E \mid B C D)$, $r(E \mid C D), r(E \mid D)$ und $r(E)$. Diese sind in den N-gramm-Tabellen unter $A B$ $C D E, B C D E, C D E, D E$ und $E$ zu finden. Da jedoch im vorherigen Schritt die Hash-Funktion die N-gramme anhand der beiden ersten Wörter verteilt hat, sind diese jetzt nicht notwendigerweise im selben Shard zu finden. Das macht das Smoothing sehr zeitaufwending. Die Lösung dafür ist, eine neue HashFunktion zu wählen, die die $\mathrm{N}$-gramme anhand der beiden letzten Wörter verteilt. Die N-gramme werden damit nach der Berechnung der relativen Häufigkeiten und vor dem Smoothing umverteilt. Diese Umverteilung ist bei 
großen Datenmengen effizienter als der wiederholte wahlfreie Zugriff auf viele Shards.

Die vier Schritte für das Erzeugen eines Sprachmodells (Vokabularerstellung, Erstellung der N-gramm-Tabellen, Berechnung der relativen Häufigkeiten und Smoothing) benötigen bei 200 Milliarden Wörtern im Eingabetext ca. einen Tag bei Verarbeitung auf 1000 Maschinen.

\section{Verwendung verteilter Sprachmodelle}

Die Verwendung sehr großer verteilter Sprachmodelle stellt eine weitere Herausforderung dar. Wahlfreier Zugriff auf Dateien auf Festplatten verbietet sich meistens aus Effizienzgründen. Wir laden deshalb jeden Shard in den Hauptspeicher einer Maschine und greifen dann auf diese über das Netzwerk zu. Dabei ist der größte Engpass die Wartezeit im Netzwerk. Ein Zugriff im Speicher einer Maschine dauert im Mikrosekundenbereich während ein Zugriff über das Netzwerk im Millisekundenbereich liegt. Die Übersetzung eines Satzes benötigt Zugriff auf ca. 1 Million N-gramme. Setzen wir einen Zugriff über das Netzwerk sehr optimistisch mit einer Millisekunde an, dann würde bei naiver Verwendung die Übersetzung des Satzes 1000 Sekunden dauern (also mehr als 16 Minuten).

Ein Zugriff pro $\mathrm{N}$-gramm ist damit viel zu ineffizient. Eine Lösung besteht darin, nicht jede Anfrage für ein N-gramm direkt zu senden, sondern diese zunächst in eine Warteschlange zu stellen, um dann später gleichzeitig auf viele $\mathrm{N}$-gramme zuzugreifen. Die Verarbeitung könnte z. B. in $20 \mathrm{Schritten}$ ablaufen, in jedem Schritt werden ca. $50.000 \mathrm{~N}$-gramme abgefragt. Dies dauert zur Zeit für alle 20 Schritte zusammen weniger als 1 Sekunde.

Unser Übersetzungssystem wechselt jetzt zwischen dem Einreihen in die Warteschlange (den "Dummy Lookups") und der eigentlichen Verwendung der N-gramme (den "True Lookups“) hin und her. Das Vorgehen ist im Suchgraphen in Abbildung 5 dargestellt. Zum Zeitpunkt $t$ werden die 3 besten Hypothesen betrachtet: AB, A, C. Jede dieser Hypothesen hat zwei mögliche Fortsetzungen, es ergeben sich sechs neue Hypothesen: AB.D, AB.C, A.BC, A.D, C.A, C.AB. Zwei davon (AB.C und A.BC) ergeben dieselbe Wort-
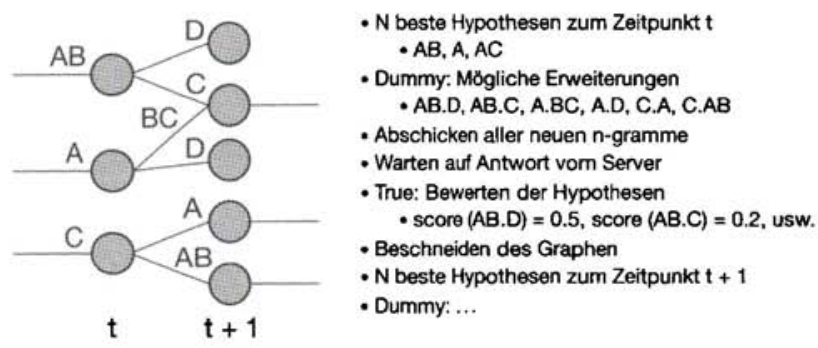

Abb. 5: Beispiel für eine Graphensuche mit verteiltem Sprachmodell und abwechselnden Dummy Lookups und True Lookups. 
sequenz. Im Dummy Lookup werden also die N-gramme für 5 neue Hypothesen in die Warteschlange eingereiht. Danach wartet das System auf die Antwort vom Server, der die Wahrscheinlichkeiten zurückliefert. Mit deren Hilfe wird nun jede Hypothese bewertet und der Graph beschnitten. Nur die besten drei verbleiben für den nächsten Schritt ( $\mathrm{ABC}, \mathrm{CA}, \mathrm{CB}$ im Beispiel). Danach beginnt der Prozess von vorn. Wiederum werden Hypothesen generiert, die Warteschlange aufgebaut, usw.

\section{Abschließende Bemerkungen}

Die Verwendung großer verteilter Sprachmodelle hat einen starken Einfluss auf die Übersetzungsqualität automatischer Systeme. Ein 5-gramm-Modell trainiert auf 150 Millionen Wörtern passt in den Speicher einer Maschine und liefert mit Google's Übersetzungssystem einen BLEU-Score von $49.13 \%$ für Arabisch-Englische Übersetzungen. Ein 5-gramm-Modell trainiert auf 200 Milliarden Wörtern belegt ca. 1 Terabyte bei Speicherung aller auftretenden N-gramme. Dies verhilft zu einem BLEU-Score von $53.10 \%$ auf den gleichen Daten. Dies ist eine enorme Steigerung. Die verteilte Verwendung des größen Sprachmodells mit Dummy Lookups und True Lookups führt dabei kaum zu Geschwindigkeitseinbußen, die Übersetzung eines Satzes geschieht in ca. einer Sekunde.

Eine der Ursachen für die hohe Qualität des großen Sprachmodells ist, dass viele der $\mathrm{N}$-gramme in zu übersetzenden Texten vom System bereits gesehen worden sind. Dies wird in der folgenden Tabelle veranschaulicht. ${ }^{6}$

\begin{tabular}{l|l|l|l|l}
\hline Training & unbekannt & 5-gramme & 4-gramme & 3-gramme \\
\hline $150 \mathrm{Mio}$ & $0.44 \%$ & $14.75 \%$ & $33.83 \%$ & $64.46 \%$ \\
\hline $200 \mathrm{Mia}$ & $0.09 \%$ & $47.52 \%$ & $72.89 \%$ & $92.20 \%$ \\
\hline
\end{tabular}

Die Tabelle zeigt Werte für zwei verschiedene Mengen von Trainingsdaten: 150 Millionen Wörter und 200 Milliarden Wörter. Die zweite Spalte gibt an, wie viele der Wörter in den Referenzübersetzungen unbekannt sind, d. h., nicht in den Trainingsdaten aufgetaucht sind. Dies sind deutlich weniger für das große Sprachmodell. Die weiteren Spalten geben an, wieviele der N-gramme bekannt sind. Die Unterschiede sind sehr groß. So „kennt“ das große Sprachmodell ungefähr dreimal so viele 5-gramme wie das kleine Sprachmodell (47.52\% gegenüber $14.75 \%$ ), kann also deutlich bessere Schätzungen für deren Wahrscheinlichkeiten angeben.

6 Alle Werte wurden auf den Arabisch-Englishen Übersetzungsdaten des Linguistic Data Consortium mit der Katalognummer LDC2005T05 gemessen. 
Sehr große N-gramm-Tabellen sind für die Forschung in einer Reihe von Gebieten nützlich. Zusätzlich zur maschinellen Übersetzung sind dies z. B. die automatische Spracherkennung, die Zeichenerkennung, Rechtschreibkorrektur und Informationsextraktion. Allerdings ist die Erstellung solcher Tabellen sehr zeit- und ressourcenaufwändig. Google plant deshalb, 5-gramm-Tabellen, erstellt aus ca. 1 Billion $\left(10^{12}\right)$ Wörtern englischer Web-Daten, für die Forschung zur Verfügung zu stellen. Damit soll allen Forschungsgruppen erlaubt werden, gemeinsam mit großen Datenmengen zu arbeiten, unabhängig von der Größe der Gruppe und ihrer Computer-Infrastruktur. Die Veröffentlichung wird ca. 6 DVDs umfassen und vom Linguistic Data Consortium vertrieben werden.

\section{Danksagung}

Vielen Dank für die gemeinsame Arbeit an der Erstellung der großen Sprachmodelle, für zahlreiche Diskussionen zum Thema, sowie für wertvolle Unterstützung bei der Erstellung dieses Artikels an Sabine Brants, Jeff Dean, Peter Dienes, Alex Franz, John Hawkins, Franz Och, Jay Ponte, Shankar Kumar, Ashok Popat, Ioannis Tsochantaridis und Peng Xu.

\section{Literatur}

Brown, P./Cocke, J./Della Pietra, S./Della Pietra, V./Jelinek, F./Lafferty, J./Mercer, R./Roossin, P. (1993): A Statistical Approach to Machine Translation. In: Computational Linguistics, 16(2), S. 263-313.

Chen, S. F./Goodman, J. T. (1998): An Empirical Study of Smoothing Techniques for Language Modeling. Technischer Report TR-10-98, Computer Science Group. Harvard University.

Dean, J./Ghemawat, S. (2004): MapReduce: Simplified Data Processing on Large Clusters. Symposium on Operating System Design and Implementation. San Francisco, CA. USA.

Ghemawat, S./Gobioff, H./Leung, S.-T. (2003): The Google File System. Symposium on Operating System Principles. Lake George, NY. USA.

Jelinek, Frederick/Mercer, Robert L. (1980): Interpolated estimation of Markov source parameters from sparse data. In Proceedings of the Workshop on Pattern Recognition in Practice. S. 381-397. Amsterdam, The Netherlands.

Koehn, P./Och, F. J./Marcu, D. (2003): Statistical Phrase-Based Translation. In Proceedings of the Human Language Technology Conference. Edmonton, Canada.

Markov, A. (1913): Russian [An example of statistical investigation in the text of Eugene Onyegin illustrating coupling of 'tests' in chains]. In: Proceedings of the Academy of Sciences. St. Petersburg, 7(VI), S. 153-162.

Och, F. J./Tillmann, C./Ney, H. (1999): Improved alignment models for statistical machine translation. In: Proc. of the Joint Conference on Empirical Methods in Natural Language Processing and Very Large Corpora. S. 20-28.

Papineni, K./Roukos, S./Ward, T./Zhu, W.-J. (2002): BLEU: a method for automatic evaluation of machine translation. In: 40th Annual Meeting of the Association for Computational Linguistics. S. 311-318. Philadelphia, PA. USA. 